Poster sessions

Abstract P198 Table 1. Percentage of patients reporting issue as causing 'quite a lot' or 'very much' distress

\begin{tabular}{|c|c|c|c|c|c|c|c|}
\hline & Issue & $\%$ & $95 \% \mathrm{Cl}$ & & Issue & $\%$ & $95 \% \mathrm{Cl}$ \\
\hline 1 & Shortness of breath & 64 & $(54-74)$ & $10=$ & The effect of your condition on your sexual life & 21 & $(12-30)$ \\
\hline 2 & Cough & 51 & $(40-61)$ & $12=$ & Changes in your ability to carry out your usual household tasks & 20 & $(12-29)$ \\
\hline 3 & Feeling tired & 49 & $(39-59)$ & $12=$ & Feeling everything is an effort & 20 & $(11-29)$ \\
\hline 4 & Dry mouth & 34 & $(24-44)$ & $14=$ & Changes in your ability to carry out your usual daily activities, e.g. bathing & 18 & $(10-26)$ \\
\hline 5 & Feeling weak & 33 & $(23-43)$ & $14=$ & Losing your independence & 18 & $(10-26)$ \\
\hline 6 & Feeling sleepy during the day & 32 & $(22-42)$ & 16 & Pain & 17 & $(9-25)$ \\
\hline 7 & Worrying about the effect of your illness on other people & 27 & $(18-37)$ & $17=$ & Feeling as if you are in a low mood & 15 & $(7-22)$ \\
\hline 8 & Problems sleeping at night & 24 & $(15-33)$ & $17=$ & Sore mouth & 15 & $(7-23)$ \\
\hline 9 & Feeling that your symptoms are not controlled & 22 & $(13-30)$ & $17=$ & Bladder problems (urinary incontinence) & 15 & $(7-23)$ \\
\hline 10 & Feeling anxious & 21 & $(12-29)$ & 20 & Feeling restless and agitated & 14 & $(6-21)$ \\
\hline
\end{tabular}

Surprisingly concerns with dry mouth, sleep and effect on sexual life were also in the 'top 10' of distressing issues: further investigation into these is suggested.

Utilisation of SPARC can highlight troublesome issues experienced by patients with Idiopathic Interstitial Lung Diseases. Addressing these concerns, which may have been otherwise unrecognised, can guide best supportive care.

\section{P199 DISCREPANCY BETWEEN SYMPTOM SEVERITY AND SELF-REPORTED PALLIATIVE CARE NEEDS IN INTERSTITIAL LUNG DISEASE PATIENTS}

S Tsim, G Chalmers; Glasgow Royal Infirmary, Glasgow, United Kingdom

\subsection{6/thoraxjnl-2013-204457.351}

Introduction Patients with interstitial lung disease (ILD) have a high symptom burden but their palliative care needs are not well reported. We hypothesised that there was an unmet need for social and palliative care input in ILD patients.

Methods 131 consecutive ILD clinic patients (September 2013January 2013) completed a questionnaire unsupervised. 6 ILD patients on home oxygen completed the same questionnaire during a home visit. The questionnaire consisted of the 15 questions included in the King's Brief Interstitial Lung Disease (K-BILD) health status questionnaire, concerning experiences in the preceding two weeks, and an additional 9 questions aimed at assessing patients' perceptions of their needs and concerns.

Results Despite reporting significant physical symptoms in the questionnaire, including breathlessness in 69\%, chest tightness in $52 \%$ and wheeze $(60 \%)$, only $10 \%$ felt that their physical needs were not being met.

Emotional and psychological symptom reporting was high, including worry about the seriousness of their lung condition (54\%), feeling "down" in 52\% and anxiety in $43 \%$. Only $32 \%$ felt in control of their lung condition, with $43 \%$ expecting to get worse and $39 \%$ thinking about the end of their life. $49 \%$ of patients worried about how their spouse or carer was coping with their condition. However, only $7 \%$ and $9 \%$ felt they needed more emotional or spiritual support respectively.

The majority of patients avoided doing things that made them breathless (64\%) and felt that their lung condition interfered with their activities of daily living (60\%) or limited them carrying things (43\%) but only $13 \%$ felt they needed more practical help.

Most patients wanted to be involved in care decisions should they become unwell $(81 \%, \mathrm{n}=110)$.
Conclusions ILD patients report significant physical and psychological symptoms, but there is a marked discrepancy between reported symptoms and the perceived need for additional support, including practical, emotional and spiritual support. The reason for this is unclear. It may relate to perceived lack of benefit of such services or to poor understanding of the questionnaire itself. Most patients wanted to be involved in care decisions if they become unwell, highlighting the importance of anticipatory care planning.

\section{P200 A QUALITATIVE EUROPEAN SURVEY OF PATIENTS PERCEPTIONS OF CURRENT MANAGEMENT OF IDIOPATHIC PULMONARY FIBROSIS}

${ }^{1} \mathrm{AM}$ Russell, ${ }^{2} \mathrm{C}$ Vancheri, ${ }^{3} \mathrm{M}$ Maronati, ${ }^{3} \mathrm{C}$ Giot; ${ }^{1}$ National Heart \& Lung Institiute, Royal Brompton, London, UK; ${ }^{2}$ University of Catania, Catania, Italy; ${ }^{3}$ InterMune IAG, Muttenz, Switzerland

\subsection{6/thoraxjnl-2013-204457.352}

Objectives Idiopathic pulmonary fibrosis (IPF) is a chronic, progressive, fibrotic lung associated with significant mortality. There has been a marked increased interest in IPF and new emerging therapies have been shown to improve either the survival or quality of life for some people with IPF. This study aimed to explore patient's perceptions of current therapy $\&$ management of IPF, specifically pirfenidone as the first approved treatment. Methods Patients diagnosed with IPF according to current criteria and prescribed pirfenidone by one of 3 European specialist ILD centres were enrolled in a qualitative survey. One-to-one in-depth interviews were conducted between September and October 2012. Results 45 Participants (71\% male; mean age 68.5 years). Mean time from diagnosis to interview 3.5 years. Post diagnosis, $68 \%$ of patients felt their knowledge about IPF severity, treatments and prognosis increased markedly, predominantly through the use of the internet. $32 \%$ of patients relied exclusively on information gained from the consultation and demonstrated a lack of understanding of the disease and its process. For all patients the transition to oxygen therapy signalled a significant change impacting upon the view of their future. $\mathrm{O} 2$ therapy was associated with social exposure of disease, often with feelings of "shame" (35\%). This impacted quality of life: "restricting activity"; "making simple tasks difficult....even talking" and was associated with impaired emotional well-being (Figure 1). There was an overwhelming lack of psychological support (79\%) as patients struggled to comprehend the disease process. Patients spontaneously identified specific approaches that could improve their 
disease experience. Pirfenidone was well tolerated and offered hope to the majority of patients (83\%). However 44\% of patients reported anxieties re continuing access.

Conclusions Post diagnosis, many patients demonstrate resourcefulness in accessing information and have realistic expectations of how to improve care. There is a need to improve the information given in the consultation to improve subsequent understanding and to increase provision of psychological support particularly when prescribing $\mathrm{O} 2$ therapy. The availability of pirfenidone was perceived by patients to offer hope and reassurance. Strategies to reduce the delay in diagnosis and standardise access to information and therapies are needed.

\section{P201 RITUXIMAB AS RESCUE THERAPY IN INTERSTITIAL LUNG DISEASE REFRACTORY TO CONVENTIONAL IMMUNOSUPPRESSION}

RR Abdullah, D Ming, GJ Keir, TM Maher, AU Wells, EA Renzoni; Interstitial Lung Disease Unit, Royal Brompton Hospital, London, UK

\subsection{6/thoraxinl-2013-204457.353}

Background Rituximab, a B cell-depleting monoclonal antibody, may offer an effective rescue therapy in a subgroup of patients with severe interstitial lung disease (ILD), progressing despite conventional immunosuppression.

Methods Retrospective assessment of 50 patients with severe, progressive ILD treated with rituximab between 2010 and 2012. This included 33 with connective tissue disease-associated ILD (CTD-ILD), 6 with fibrotic hypersensitivity pneumonitis, 3 with likely drug-induced ILD, 2 with desquamative interstitial pneumonia (DIP) and the rest with miscellaneous ILD patterns, excluding idiopathic pulmonary fibrosis. At the time of rituximab treatment, mean FVC was $49.1 \%(+17.6)$ and DLco $25.5 \%(+9.9)$. Four patients were mechanically ventilated. Prior to rituximab, all patients except one had received immunosuppressive treatment, including IV cyclophosphamide in 44 patients. Change in pulmonary function tests, as compared to pre-rituximab levels, was assessed at six to twelve months posttreatment and analysed by Wilcoxon signed rank test. Categorical trends (improvement, stability, deterioration) before and after treatment were defined using either $\geq 10 \%$ change in forced vital capacity (FVC) or $\geq 15 \%$ change in diffusing capacity for carbon monoxide (DLco) as threshold values.

Results In the six to twelve months following rituximab treatment, a median improvement in FVC of 5.7\% $(\mathrm{p}<0.01)$ and stability of DLco $(p<0.01)$ was observed. This was in contrast to a median decline in FVC and DLco of $14.6 \%$ and $18.8 \%$ respectively, in the six to twelve months prior to rituximab therapy $(\mathrm{p}<$ 0.01). Patients with CTD-ILD were most represented in this cohort and were more likely to improve or stabilise following Rituximab (28/33), than those with non CTD-related ILD (8/17) ( $\mathrm{p}=0.008$, Fisher exact test). However, of the four patients requiring invasive ventilation, improvement to extubation was observed in three patients with non CTD-ILD (one DIP, one acute interstitial pneumonia, one unclassifiable ILD). Two patients developed serious infections (pneumonia) requiring hospitalisation following rituximab, and ten patients died, all from progression of underlying ILD, a median of 5.1 months after treatment.

Conclusions Rituximab may offer a safe and effective therapeutic intervention in a subgroup of patients with severe, progressive ILD unresponsive to conventional immunosuppression. Future prospective, controlled trials are warranted to validate these findings.

\section{P202 A SURVEY OF ILD EXPERTISE AVAILABILITY AND HIGH RESOLUTION COMPUTER TOMOGRAPHY (HRCT) PROTOCOLS USED IN PATIENTS WITH INTERSTITIAL LUNG DISEASE (ILD) ACROSS HOSPITALS IN ENGLAND}

H Balata, A Ashish, I Aziz; Royal Albert Edward Infirmary, Wigan, England, UK

\subsection{6/thoraxjnl-2013-204457.354}

Introduction and aims ILD is increasingly being recognised as complex condition necessitating a multi-disciplinary approach to diagnosis and management requiring availability of a clinician and a radiologist with declared interest in ILD. HRCT is the imaging of choice used for assessment of ILD. The BTS ILD guidelines do recommend a "standard" HRCT protocol to be used in diagnosis of patients with ILD. It is however not known if there is a uniform availability of expertise within different centres in England and if "standard "HRCT protocol as recommended is being followed.

Method A questionnaire was handed to radiologists with special interest in thoracic imaging working in different hospitals sites in England, at an ILD radiology conference. Questionnaires enquired about availability of ILD services and HRCT scanning technique used at their establishment.

Results Of the 150 questionnaires, 100 were returned for analysis. There were responses from 39 teaching hospitals and 61 district general hospitals (DGH).

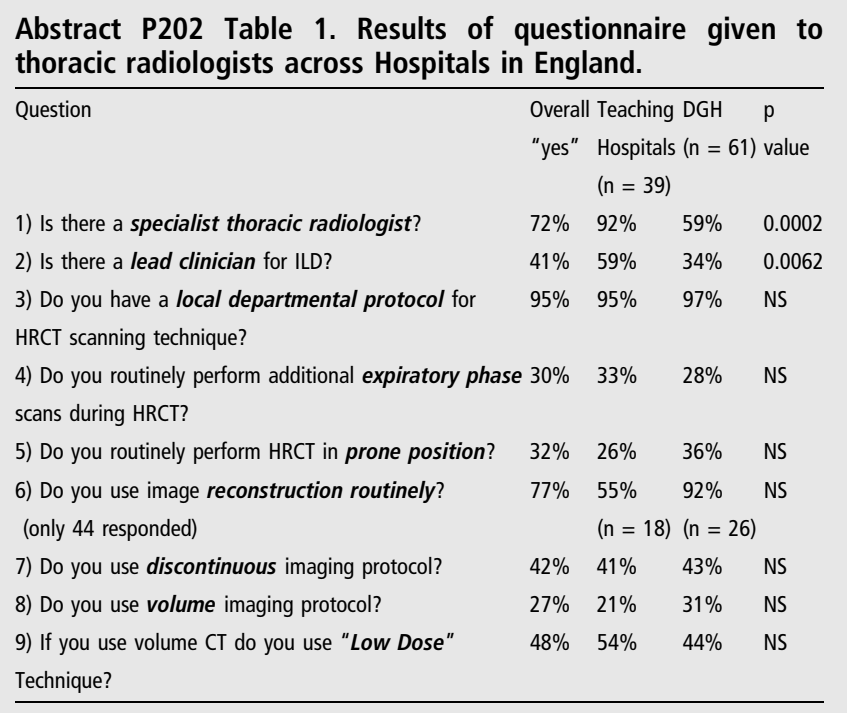

Conclusions Despite increasing focus on ILD as a sub-speciality, there is still a significant difference in the provision of expert care within district general hospitals in UK for patients with ILD. This may affect the quality of care provided with potential to variability of care standards.

The "standard protocol" for HRCT techniques as specified by BTS is not being followed in England. Despite recommendations from BTS, aspects of HRCT scanning technique applied were variable and influenced by local preferences and expertise. This may lead to differences in scan interpretation, diagnosis and outcomes. This gap in provision of care and variability of techniques should be bridged to ensure uniformity of care and outcomes.

REFERENCES

1. NICE guidelines, June 2013; CG163 2: Thorax; 63 (Suppl V); v1-v58 